\title{
Numerical Simulation of Condensation of Bubbles under Microgravity Conditions by Moving Mesh Method in the Double-staggered Grid
}

\author{
Lin Pu • Huixiong Li · Jianfu Zhao • Tingkuan Chen
}

Received: 14 February 2009 / Accepted: 3 June 2009 / Published online: 20 June 2009

(C) Springer Science + Business Media B.V. 2009

\begin{abstract}
A numerical 2D method for simulation of two-phase flows including phase change under microgravity conditions is presented in this paper, with a level set method being coupled with the moving mesh method in the double-staggered grid systems. When the grid lines bend very much in a curvilinear grid, great errors may be generated by using the collocated grid or the staggered grid. So the double-staggered grid was adopted in this paper. The level set method is used to track the liquid-vapor interface. The numerical analysis is fulfilled by solving the Navier-Stokes equations using the SIMPLER method, and the surface tension force is modeled by a continuum surface force approximation. A comparison of the numerical results obtained with different numerical strategies shows that the doublestaggered grid moving-mesh method presented in this paper is more accurate than that used previously in the collocated grid system. Based on the method presented in this paper, the condensation of a single bubble in the cold water under different level of gravity is simulated. The results show that the condensation process under the normal gravity condition is different from the condensation process under microgravity conditions.
\end{abstract}

L. Pu $\cdot$ H. Li $(\bowtie) \cdot$ T. Chen

State Key Laboratory of Multiphase Flow in Power

Engineering, Department of Thermal Engineering,

School of Energy and Power Engineering,

Xi'an Jiaotong University, Xi'an 710049, China

e-mail: huixiong@mail.xjtu.edu.cn

H. Li · J. Zhao

National Microgravity Laboratory, Institute of Mechanics,

Chinese Academy of Sciences, 15 Beisihuan Xilu,

Beijing, 10080, China
The whole condensation time is much longer under the normal gravity than under the microgravity conditions.

Keywords Condensation • Moving mesh method • Double-staggered grid $\cdot$ Microgravity

\section{Nomenclature}

$A_{E} \quad$ coefficient respect to the point $(i+1, j)$

$A_{N} \quad$ coefficient respect to the point $(i, j+1)$

$A_{p} \quad$ coefficient respect to the point (i,j)

$A_{S} \quad$ coefficient respect to the point $(i, j-1)$

$A_{W} \quad$ coefficient respect to the point $(i-1, j)$

$\mathrm{C}_{\mathrm{P}} \quad$ specific heat at constant pressure $\left(\mathrm{J} \cdot \mathrm{kg}^{-1} \cdot \mathrm{K}^{-1}\right)$

$\mathrm{F}_{\text {sur }} \quad$ surface tension force $\left(\mathrm{N} \cdot \mathrm{m}^{-2}\right)$

$\mathrm{F}_{\mathrm{X}} \quad$ body force in the $\mathrm{x}$ direction $\left(\mathrm{N} \cdot \mathrm{m}^{-2}\right)$

$\mathrm{F}_{\mathrm{y}} \quad$ body force in the $\mathrm{y}$ direction $\left(\mathrm{N} \cdot \mathrm{m}^{-2}\right)$

$\mathrm{h}_{\mathrm{lg}} \quad$ latent heat of heat transfer $\left(\mathrm{J} \cdot \mathrm{kg}^{-1}\right)$

$\mathrm{h} \quad$ grid spacing $(\mathrm{m})$

$\mathrm{H}$ heaviside function

$\mathrm{J} \quad$ Jacobian of transformation

$\mathrm{k} \quad$ thermal conductivity $\left(\mathrm{W} \cdot \mathrm{m}^{-1} \cdot \mathrm{K}^{-1}\right)$

$\vec{m} \quad$ mass flux vector $\left(\mathrm{kg} \cdot \mathrm{s}^{-1} \cdot \mathrm{m}^{-2}\right)$

$\mathrm{P} \quad$ pressure $(\mathrm{Pa})$

$\mathrm{t}$ time (s)

$\mathrm{T}$ temperature (K)

$\mathrm{T}_{1} \quad$ initial temperature of the water $(\mathrm{K})$

$\mathrm{T}_{\mathrm{V}} \quad$ initial temperature of the vapor $(\mathrm{K})$

$\mathrm{T}_{\mathrm{sat}} \quad$ saturation temperature $(\mathrm{K})$

$\Delta T \quad$ temperature difference $(\mathrm{K})$

$\mathrm{u} \quad$ velocity parallel to $\mathrm{x}\left(\mathrm{m} \cdot \mathrm{s}^{-1}\right)$

$\mathrm{v} \quad$ velocity parallel to $\mathrm{y}\left(\mathrm{m} \cdot \mathrm{s}^{-1}\right)$

$\dot{x} \quad$ mesh velocity in $\mathrm{x}$ direction $\left(\mathrm{m} \cdot \mathrm{s}^{-1}\right)$

$\dot{y} \quad$ mesh velocity in y direction $\left(\mathrm{m} \cdot \mathrm{s}^{-1}\right)$

$\alpha, \beta, \gamma \quad$ geometry coefficients 
$\varepsilon \quad$ curvilinear coordinate $(\mathrm{m})$

$\eta \quad$ curvilinear coordinate $(\mathrm{m})$

$\Phi \quad$ level set function

$\dot{\Phi} \quad$ source for the latent heat due to phase change $\left(\mathrm{J} \cdot \mathrm{s}^{-1}\right)$

$\mu \quad$ viscosity $\left(\mathrm{kg} \cdot \mathrm{m}^{-1} \cdot \mathrm{s}^{-1}\right)$

$\rho \quad$ density $\left(\mathrm{kg} \cdot \mathrm{m}^{-3}\right)$

$\sigma \quad$ surface tension $\left(\mathrm{N} \cdot \mathrm{m}^{-1}\right)$

\section{Subscript}

1 liquid

$\mathrm{v}$ vapor

$\varepsilon \quad$ partial differentiation with respect to $\varepsilon$

$\eta \quad$ partial differentiation with respect to $\eta$

$\tau$ artificial time

\section{Superscript}

$$
\begin{array}{ll}
\mathrm{n} & \text { time step } \mathrm{n} \\
\mathrm{n}+1 & \text { time step } \mathrm{n}+1
\end{array}
$$

\section{Introduction}

In a collocated grid system, velocity $\mathrm{u}, \mathrm{v}$ and pressure $\mathrm{p}$ are all stored at the grid nodes, as shown in Fig. 1. The storage memory and the computational time are reduced considerably due to its simplicity. The cell face velocities used in the continuity equation are calculated by interpolating the velocities at two neighbor nodes on the either side of the face. However, the check board oscillation of pressure may be generated due to the poor pressure-velocity coupling.

The momentum interpolation method was first proposed by Rhie and Chow (1983) to solve the check-

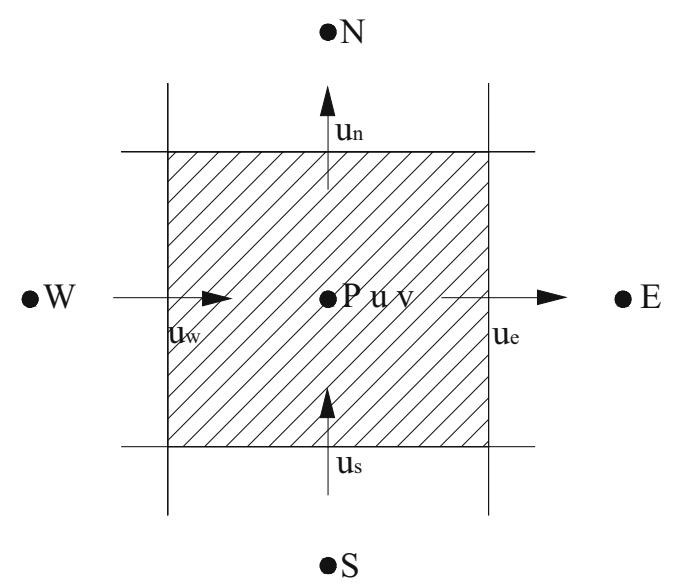

Fig. 1 Schematic of a collocated grid board pressure problem. In other words, the velocity values required for continuity equation are interpolated from the momentum equations, rather than linear interpolation from the adjacent nodal values. Choi revised (Choi 1999) Rhie and Chow's scheme, making it time step size independent. In order to make Rhie and Chow scheme underrelaxation factor-independent, Majumdar (1988) suggested a new method. Although the momentum interpolation method can solve the pressure-velocity decoupling efficiently, yet unreasonable results may be achieved when the pressure distributions are fundamentally different from the linear distribution in a specified direction (Miller and Schmidt 1988).

In the staggered grid, velocity $\mathrm{u}, \mathrm{v}$ and pressure $\mathrm{p}$ are stored in three different sets of grids, as shown in Fig. 2. Velocity $u$ is stored on the east and the west faces of the pressure control volume, and velocity $\mathrm{v}$ is stored on the north and the south faces of the pressure control volume. There are half a control volume width apart in each coordinate. In the staggered grid, the velocities and pressure are coupled well and the checkboard oscillation of pressure will not occur.

It should be noted that when the grid lines bend very much, such as the angle of bending maybe more than $90^{\circ}$, as show in Fig. 3, great errors maybe generated by using the staggered grid. $\mathrm{P}_{1}, \mathrm{U}_{2}$ and $\mathrm{V}_{1}$ can be redeemed as $\mathrm{P}, \mathrm{U}$ and $\mathrm{V}$ in staggered grid. When the computation is changed from point $\mathrm{P}_{1}\left(\mathrm{i}^{\prime}, \mathrm{j}^{\prime}\right)$ to point $\mathrm{P}_{1}\left(\mathrm{i}^{\prime}, \mathrm{j}^{\prime}\right)$, the velocity $\mathrm{u}$ and $\mathrm{v}$ make no contribution to mass flux for the point $\mathrm{P}_{1}\left(\mathrm{i}^{\prime}, \mathrm{j}^{\prime}\right)$ (Shklyar and Arbel 2003). It is the major cause of unfitness in using the staggered grid in curvilinear grid system.

However, the problem can be rectified by using the double-staggered grid system, in which, all variables have two sets. $P_{1}, U_{1}$ and $V_{2}$ are already in staggered grid, while $P_{2}, U_{2}$ and $V_{1}$ are the added variables in the double-staggered grid. Although the velocity $\mathrm{U}_{1}$ and $\mathrm{V}_{2}$ contribute nothing to the point $\mathrm{P}_{1}\left(\mathrm{i}^{\prime}, \mathrm{j}^{\prime}\right)$, they contributed greatly to the cell $\mathrm{P}_{2}\left(\mathrm{i}^{\prime}, \mathrm{j}^{\prime}\right)$. Details about the double-staggered grid can be found in literature (Yuan et al. 2008). Generally, the double-staggered grid system is more suitable for the curvilinear grid than the normal staggered grid.

Moving mesh method is employed to increase the numerical accuracy in this paper. The meshes changes continuously in space and time in order to meet the dynamic changes of a time-evolving solution. Moving mesh method can also calculate the heat transfer more accurate than a method based on uniform grids with the same number of points. More information of moving mesh method can be seen in the literature $(\mathrm{Wu}$ and Dhir 2007). 


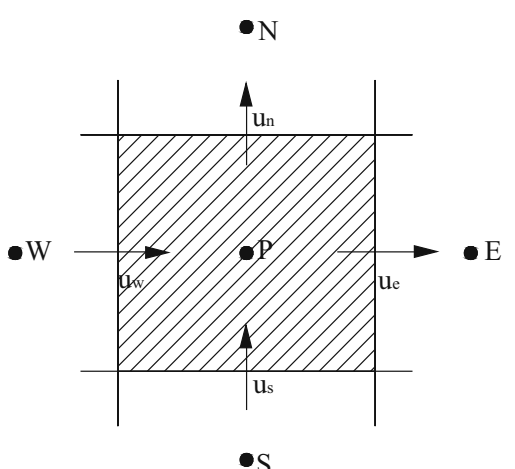

(a)

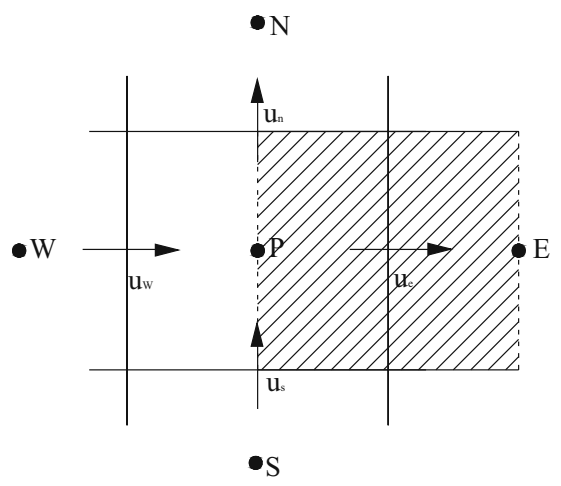

(b)

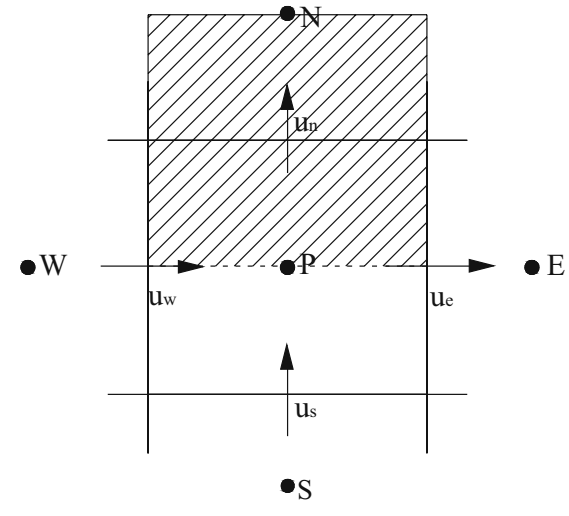

(c)

Fig. 2 Schematic of a staggered grid (a) main control volume (b) u control volume (c) v control volume

When the grid lines bend too much, great errors may be generated by using the collocated grid or the staggered grid. So in this paper, moving mesh method is coupled with the double-staggered grid to solve this problem.

Based on the method presented in this paper, the condensation of a single bubble in the cold water under different level of gravity is simulated. The great temperature difference between steam and cold water will lead to rapid condensation as soon as two phase fluids meet. In the nuclear power plant, steam bubble forms

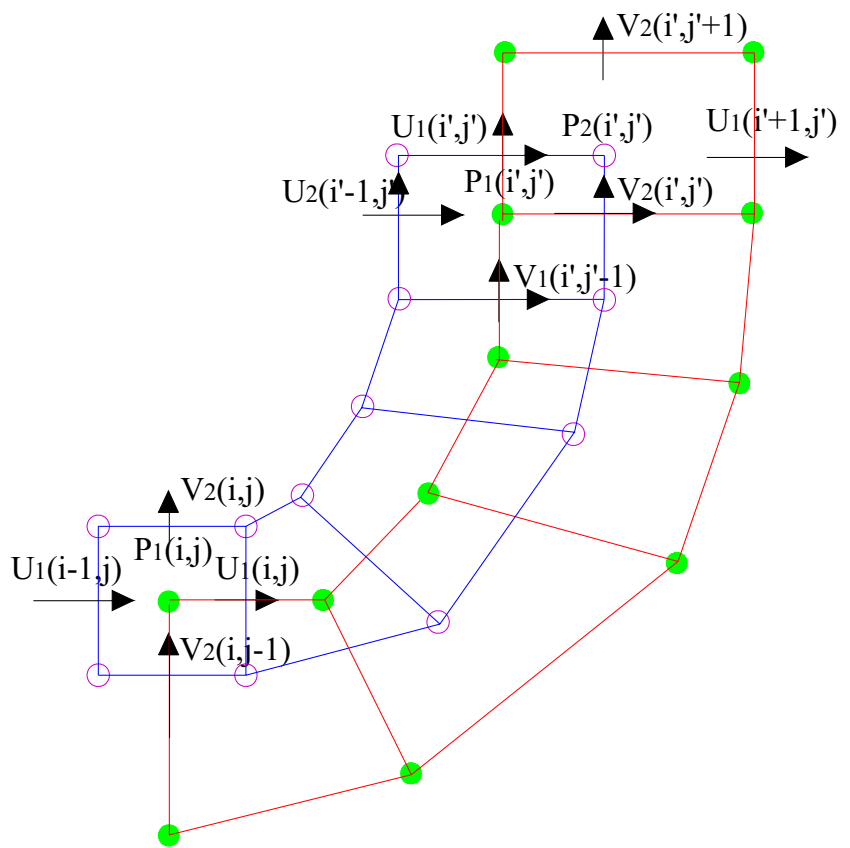

Fig. 3 Schematic of a double-staggered grid and collapses under some conditions. So the study of the condensation of the bubble in the cold water has great significances in practice.

\section{Governing Equations}

In a curvilinear coordinate system, all the variables may have two domains, respectively, a physical domain and a computational domain. The grid cells are usually irregular in physical domain, while the grid cells are all uniform in the computational domain, as shown in Fig. 4.

All the equations must be transformed from the orthogonal coordinates into the curvilinear coordinates. Geometry coefficients, $\alpha, \beta, \gamma$, and the jacobian of transformation, $\mathrm{J}$ may be used in the following equations. So they must be predefined as

$\alpha=x_{\eta}^{2}+y_{\eta}^{2}, \beta=x_{\varepsilon} x_{\eta}+y_{\varepsilon} y_{\eta}$,

$\gamma=x_{\varepsilon}^{2}+y_{\varepsilon}^{2}, J=x_{\varepsilon} y_{\eta}-x_{\eta} y_{\varepsilon}$

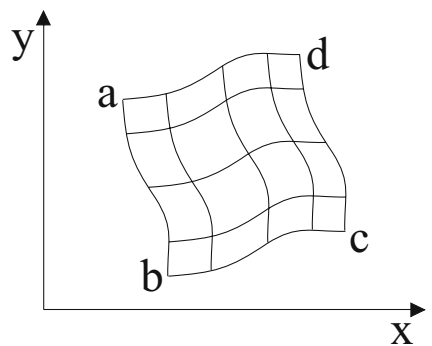

(a)

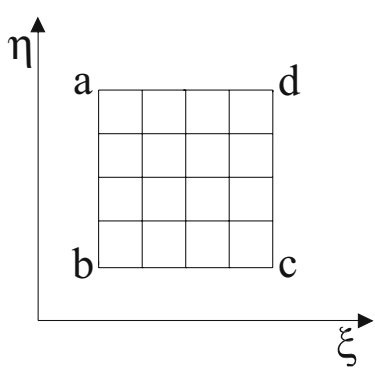

(b)
Fig. 4 Comparison of the physical domain and the computational domain (a) physical domain (b) computational domain 
Momentum equations can be expressed in the adaptive curvilinear coordinates as follows

$$
\begin{array}{r}
\rho\left[\begin{array}{c}
J \frac{\partial u}{\partial t}+u_{\varepsilon}\left[(u-\dot{x}) y_{\eta}-(v-\dot{y}) x_{\eta}\right] \\
+u_{\eta}\left[-(u-\dot{x}) y_{\varepsilon}+(v-\dot{y}) x_{\varepsilon}\right]
\end{array}\right] \\
=-y_{\eta} p_{\varepsilon}+y_{\varepsilon} p_{\eta}+\left[\frac{\mu}{J}\left(\alpha u_{\varepsilon}-\beta u_{\eta}\right)\right]_{\varepsilon} \\
+\left[\frac{\mu}{J}\left(\gamma u_{\eta}-\beta u_{\varepsilon}\right)\right]_{\eta}+F_{x} \\
\rho\left[\begin{array}{l}
J \frac{\partial v}{\partial t}+v_{\varepsilon}\left[(u-\dot{x}) y_{\eta}-(v-\dot{y}) x_{\eta}\right]+ \\
v_{\eta}\left[-(u-\dot{x}) y_{\varepsilon}+(v-\dot{y}) x_{\varepsilon}\right]
\end{array}\right] \\
=-x_{\varepsilon} p_{\eta}+x_{\eta} p_{\varepsilon}+\left[\frac{\mu}{J}\left(\alpha v_{\varepsilon}-\beta v_{\eta}\right)\right]_{\varepsilon} \\
+\left[\frac{\mu}{J}\left(\gamma v_{\eta}-\beta v_{\varepsilon}\right)\right]_{\eta}+F_{y}
\end{array}
$$

Where $F_{x}$ and $F_{y}$ is the components of the body force. Two kinds of forces are encountered in the present study, i.e., the microgravity and the surface tension force. We use the continuum approach to represent the surface tension force as follows

$$
F_{\text {sur }}=\sigma \kappa \nabla H
$$

Where $\kappa$ is the interfacial curvature, defined as below $\kappa=\nabla \cdot(\nabla \Phi /|\nabla \Phi|)$

$\mathrm{H}$ is the heaviside function, defined as follows

$$
H(\Phi)= \begin{cases}0 & \Phi<-1.5 h \\ 0.5+\Phi /(3 h)+\sin [2 \pi \Phi /(3 h)] / 2 \pi & |\Phi| \leq 1.5 h \\ 1 & \Phi 1.5 h\end{cases}
$$

Level set method is employed to track the liquidvapor interface. Level set equation and the reinitializa- tion equation are also written in body-fitted curvilinear coordinates as below

$$
\begin{aligned}
& J \frac{\partial \Phi}{\partial t}+\Phi_{\varepsilon}\left[(\hat{u}-\dot{x}) y_{\eta}-(\hat{v}-\dot{y}) x_{\eta}\right] \\
& +\Phi_{\eta}\left[-(\hat{u}-\dot{x}) y_{\varepsilon}+(\hat{v}-\dot{y}) x_{\varepsilon}\right]=0 \\
& \frac{\partial \Phi}{\partial \tau}=\operatorname{sign}\left(\varphi_{0}\right)\left[1-J^{-1} \sqrt{\alpha \varphi_{\varepsilon}^{2}+\gamma \varphi_{\eta}^{2}-2 \beta \varphi_{\varepsilon} \varphi_{\eta}}\right]
\end{aligned}
$$

Where $\hat{u}$ and $\hat{v}$ are the interfacial velocities, defined as

$\hat{u}=u+\frac{k}{J h_{\lg } \rho}\left(y_{\eta} T_{\varepsilon}-y_{\varepsilon} T_{\eta}\right)$,

$\hat{v}=v+\frac{k}{J h_{\lg } \rho}\left(-x_{\eta} T_{\varepsilon}+x_{\varepsilon} T_{\eta}\right)$.

Continuity equation is as below

$\frac{1}{J}\left[\begin{array}{l}{\left[(u-\dot{x}) y_{\eta}-(v-\dot{y}) x_{\eta}\right]} \\ +\left[-(u-\dot{x}) y_{\varepsilon}+(v-\dot{y}) x_{\varepsilon}\right]\end{array}\right]=\left(\frac{1}{\rho_{l}}-\frac{1}{\rho_{v}}\right) \vec{m} \cdot \nabla H$

Where $\vec{m}$ is vapor-condensation-rate vector, which is defined as

$\vec{m}=\frac{k \nabla T}{h_{\mathrm{lg}}}$

The energy equation is as follows

$\begin{cases}\rho c_{p l}\left[\begin{array}{ll}J \frac{\partial T}{\partial t}+T_{\varepsilon}\left[(u-\dot{x}) y_{\eta}-(v-\dot{y}) x_{\eta}\right]+ \\ T_{\eta}\left[-(u-\dot{x}) y_{\varepsilon}+(v-\dot{y}) x_{\varepsilon}\right]\end{array}\right] & \\ =\left[\frac{k}{J}\left(\alpha T_{\varepsilon}-\beta T_{\eta}\right)\right]_{\varepsilon}+\left[\frac{k}{J}\left(\gamma T_{\eta}-\beta T_{\varepsilon}\right)\right]_{\eta}+\dot{\Phi} & H>0 \\ T=T_{\text {sat }} & H=0\end{cases}$

Where $\dot{\Phi}$ represents the contributions of the latent heat of condensation to the energy equation, defined as below

$\dot{\Phi}=\left[\frac{\partial(\rho \cdot \Delta L)}{\partial t}+\frac{\partial(\rho \cdot u \cdot \Delta L)}{\partial x}+\frac{\partial(\rho \cdot v \cdot \Delta L)}{\partial y}\right]$

Here $\Delta L$ is the heat released by the condensation, as follows

$\Delta L=h_{\lg } \cdot[1-H]$
Table 1 The comparison of the dimensionless terminal velocities between the results from the collocated grid and Ryskin\&Leal's

\begin{tabular}{llllll}
\hline Case & $\mathrm{N}_{\mathrm{f}}$ & $\mathrm{E}_{\mathrm{k}}$ & Ryskin\&Leal & $\begin{array}{l}\text { Results from a } \\
64 \times 128 \text { mesh of } \\
\text { the present work }\end{array}$ & $\begin{array}{l}\text { Results from a } \\
128 \times 256 \text { mesh of } \\
\text { the present work }\end{array}$ \\
\hline 1 & 2.04 & 20.7 & 0.49 & 0.53 & 0.51 \\
2 & 8.66 & 1.26 & 1.16 & 1.20 & 1.18 \\
\hline
\end{tabular}


Table 2 The comparison of the dimensionless terminal velocities between the results from the double-staggered grid and Ryskin\&Leal's

\begin{tabular}{llllll}
\hline Case & $\mathrm{N}_{\mathrm{f}}$ & $\mathrm{E}_{\mathrm{k}}$ & Ryskin\&Leal & $\begin{array}{l}\text { Results from a } \\
64 \times 128 \text { mesh of } \\
\text { the present work }\end{array}$ & $\begin{array}{l}\text { Results from a } \\
128 \times 256 \text { mesh of } \\
\text { the present work }\end{array}$ \\
\hline 1 & 2.04 & 20.7 & 0.49 & 0.52 & 0.50 \\
2 & 8.66 & 1.26 & 1.16 & 1.19 & 1.17 \\
\hline
\end{tabular}

\section{Numerical Experiments}

Bubble rising in a liquid is used as a test for the method presented in this paper. Starting from a perfectly circular bubble which is initially at rest, the upward motion of the bubble in a gravitational field is studied by tracking the liquid-vapor interface. To prevent the rising bubble from escaping out of the computational domain, the computational domain is moved with the bubble rise speed. The bubble will reach a steady state until the wall velocity is equal to the bubble rise speed. In this test, all the governing equations are changed into Nondimensional. Lengths are scaled by the bubble radius $\mathrm{R}$, and velocities by $\sqrt{g R}$. Other dimensionless parameters are defined as:

$\mathrm{N}_{\mathrm{f}}=\frac{\rho_{l} \mathrm{~g}^{1 / 2} R^{3 / 2}}{\mu_{l}}, \mathrm{E}_{\mathrm{k}}=\frac{\rho_{l} g R^{2}}{\sigma}$

The computational domain is chosen as a large rectangle domain, $6 \mathrm{R}$ in width and $12 \mathrm{R}$ in height. The properties of the vapor and liquid are: $\rho_{l} / \rho_{v}=1000, \mu_{l} / \mu_{v}=100$.

Table 1 shows the comparison of the dimensionless terminal velocities of the rising bubble between results from the collocated and the Ryskin\&Leal's (Ryskin and Leal 1984). For the results from the $64 \times 128$ mesh, the deviations from Ryskin\&Leal's are less than $10 \%$. For the results from a $128 \times 256$ mesh, the deviations from Ryskin\&Leal's are less than $5 \%$.

Table 2 shows the comparison of the dimensionless terminal velocities of the rising bubble between results from the double-staggered grid and the Ryskin\&Leal's. For the results from the $64 \times 128$ mesh, the deviations from Ryskin\&Leal's are less than $6 \%$. For the results from a $128 \times 256$ mesh, the deviations from Ryskin\&Leal's are less than $2 \%$.

As a result, the comparisons show the results from the double-staggered grid are more accurate than the results from the collocated grid. Figure 5 shows the bubble shape at the steady state for $\mathrm{N}_{\mathrm{f}}=2.04$ and $\mathrm{E}_{\mathrm{k}}=20.7$ from the double-staggered grid. Figure 6 shows the bubble shape at the steady state for $\mathrm{N}_{\mathrm{f}}=8.66$ and $E_{k}=1.26$ from the double-staggered grid.

\section{Single Bubble Condensating in the Cold Water}

\section{Physical Model}

The model presented in this paper is for computing a $2 \mathrm{~d}$ and symmetric left-right incompressible two-phase flows, as shown in Fig. 7. The bubble initially located

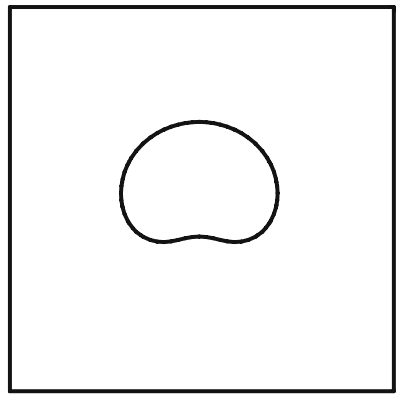

(a)

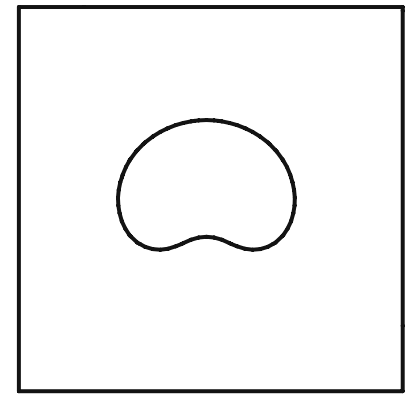

(b)

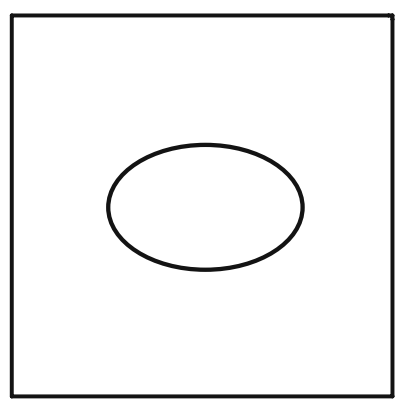

(a)

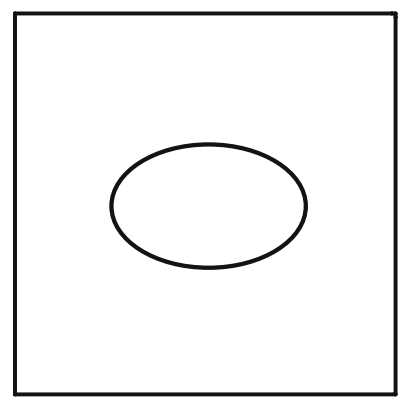

(b)

Fig. 5 Bubble shape at the steady state for $\mathrm{N}_{\mathrm{f}}=2.04$ and $\mathrm{E}_{\mathrm{k}}=$ 20.7 from the double-staggered grid (a) the results from a $64 \times$ 128 grid $(\mathbf{b})$ the results from a $128 \times 256$ grid

Fig. 6 Bubble shape at the steady state for $N_{f}=8.66$ and $E_{k}=$ 1.26 from the double-staggered grid (a) the results from a $64 \times$ 128 grid (b) the results from a $128 \times 256$ grid 


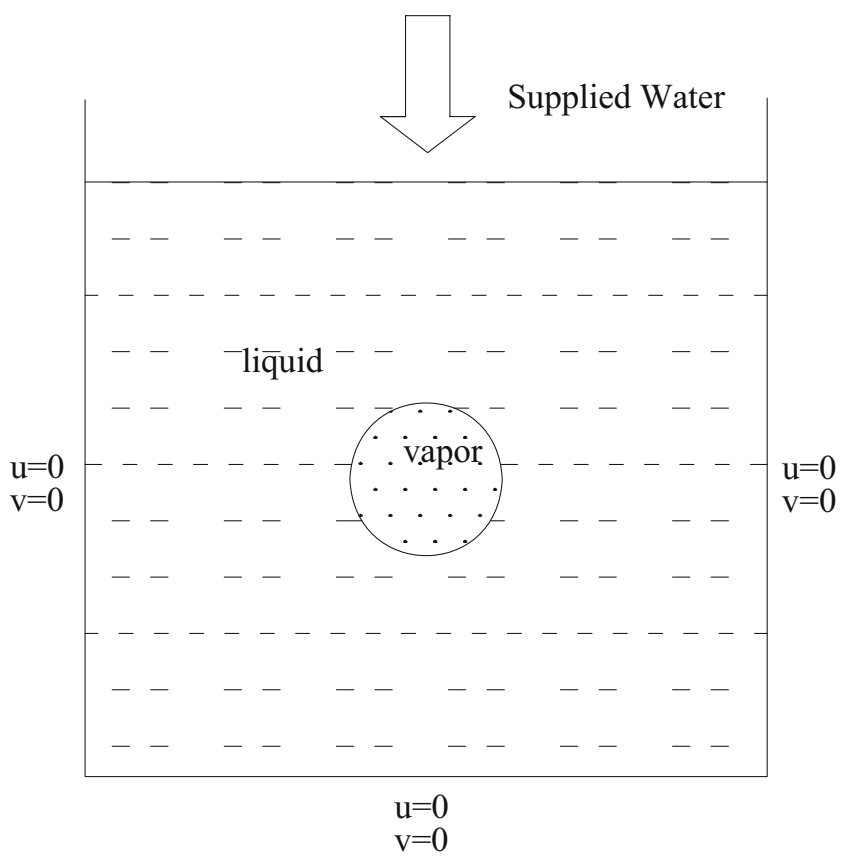

Fig. 7 Physical model

at $(0.1,0.1)$ in the domain of a size $(0.2,0.2)$. The initial radius of the bubble is 0.02 . The temperature of the cold liquid is $293.15 \mathrm{~K}$ and the vapor is $373.15 \mathrm{~K}$.

Equation 1-5 are nondimensionalized using the characteristic length, time, velocity and temperature scales $\mathrm{l}_{0}, \mathrm{t}_{0}, \mathrm{u}_{0}$ and $\theta$, respectively, as below

$$
\begin{aligned}
& l_{0}=\sqrt{\frac{\sigma}{g\left(\rho_{l}-\rho_{v}\right)}}, \quad t_{0}=\left(\frac{\sigma}{g^{3}\left(\rho_{l}-\rho_{v}\right)}\right)^{1 / 4}, \\
& u_{0}=\left(\frac{\sigma g}{\left(\rho_{l}-\rho_{v}\right)}\right)^{1 / 4}, \theta=\frac{T-T_{l}}{T_{v}-T_{l}}
\end{aligned}
$$

Fig. 8 Grid distributions at the time intervals of 0,20 and 40 ms under the normal gravity condition $(\mathbf{a}-\mathbf{c})$
Dimensionless parameters are defined as

$$
\begin{gathered}
\mathrm{N}_{\mathrm{f}}=\frac{\rho_{l} u_{0} l_{0}}{\mu_{l}}, \quad \mathrm{E}_{\mathrm{k}}=\frac{\rho_{l} u_{0}^{2} l_{0}}{\sigma}, \\
P e=\frac{\rho_{l} c_{p l} u_{0} l_{0}}{k_{l}}, \quad J a=\frac{c_{p l} \Delta T}{h_{l g}}
\end{gathered}
$$

The dimensionless parameters are evaluated as

$$
\begin{aligned}
\rho_{l} / \rho_{v} & =1000, \mu_{l} / \mu_{v}=83.7, \mathrm{~N}_{\mathrm{f}}=443.9, \mathrm{E}_{\mathrm{k}}=1.0, \\
\mathrm{Pe} & =3112.5 \text { and } \mathrm{Ja}=148.2 \text { for } \Delta T=80 K .
\end{aligned}
$$

\section{Boundary Conditions}

In this study, all of the boundaries except the top of the domain are described by

$u=0, v=0$

The condition on the top of the domain is described by

$\frac{\partial u}{\partial x}=0, \quad \frac{\partial v}{\partial y}=0$

The condition on the top of the domain should be revised to make the volume of water supplied equal to the volume of gas reduced due to condensation in one time layer.

\section{Moving Mesh}

\section{Mesh Generation Procedure}

The mesh-generation procedure given by Ceniceros and Hou (2001) is utilized in this article. The mesh generation equations in the computational domain are simplified as follows:

$x_{\tau}=\nabla^{\prime}\left(w \nabla^{\prime} x\right), y_{\tau}=\nabla^{\prime}\left(w \nabla^{\prime} y\right)$

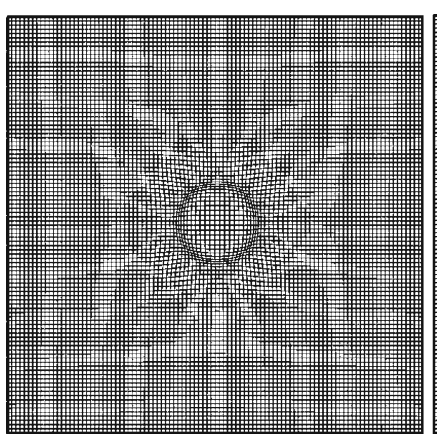

(a) $\mathrm{t}=1 \mathrm{~ms}$

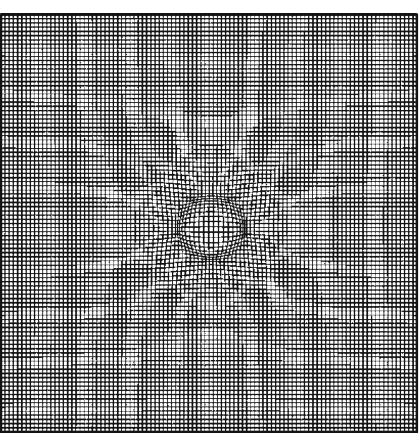

(b) $\mathrm{t}=20 \mathrm{~ms}$

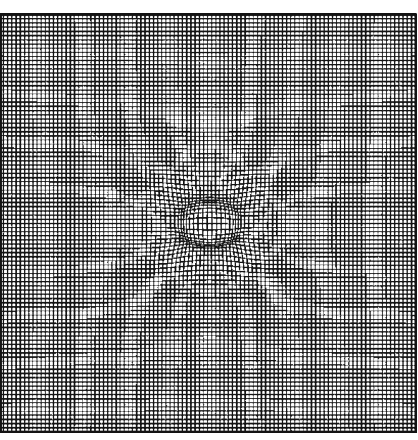

(c) $\mathrm{t}=40 \mathrm{~ms}$ 


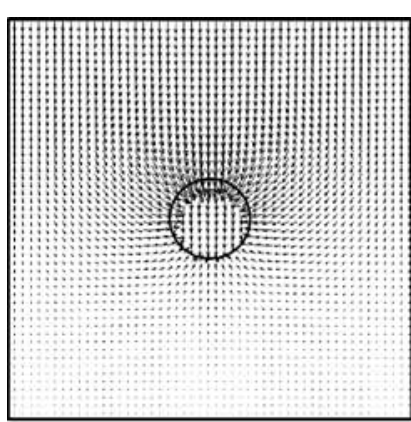

(a) $\mathrm{t}=1 \mathrm{~ms}$

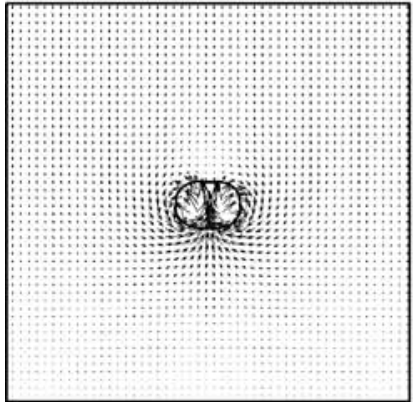

(c) $\mathrm{t}=40 \mathrm{~ms}$

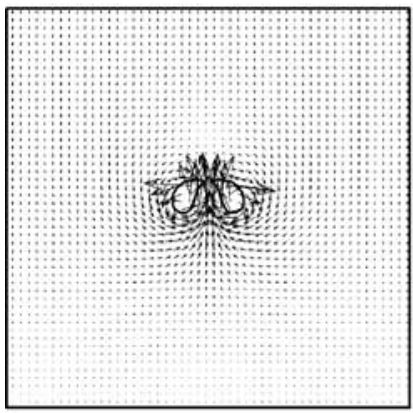

(e) $\mathrm{t}=80 \mathrm{~ms}$

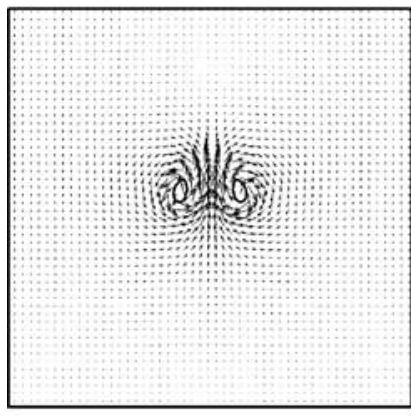

(g) $\mathrm{t}=120 \mathrm{~ms}$

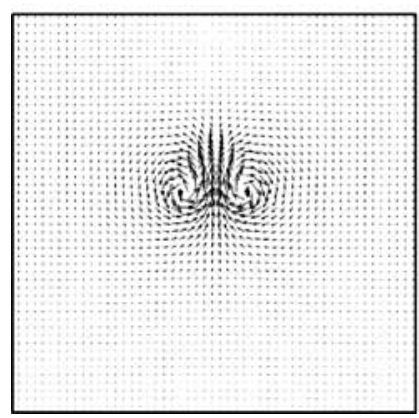

(i) $\mathrm{t}=160 \mathrm{~ms}$

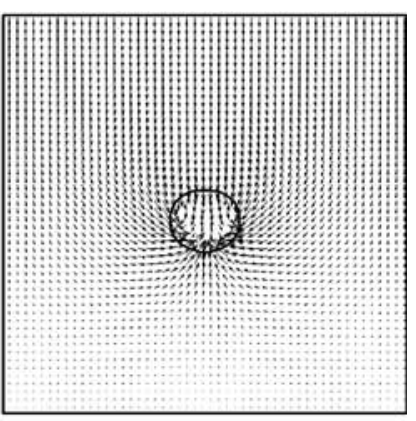

(b) $\mathrm{t}=20 \mathrm{~ms}$

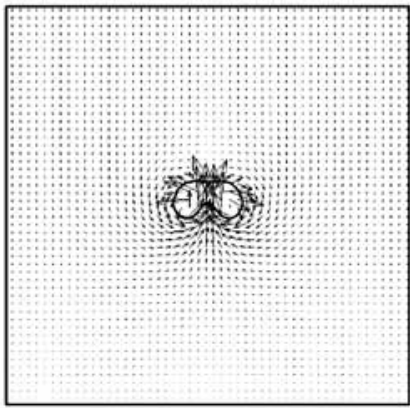

(d) $\mathrm{t}=60 \mathrm{~ms}$

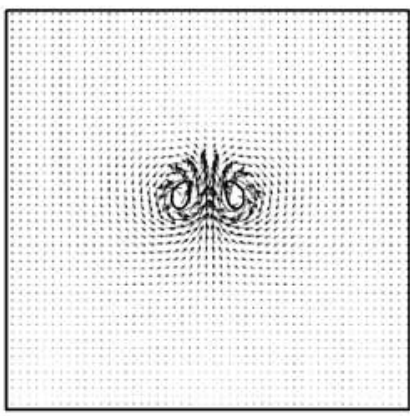

(f) $\mathrm{t}=100 \mathrm{~ms}$

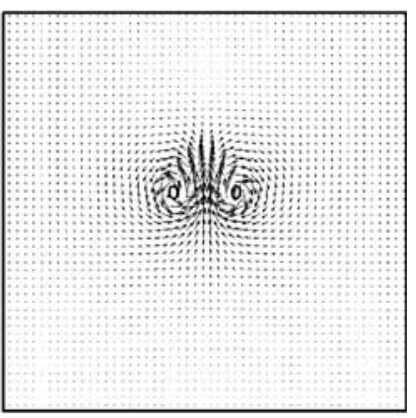

(h) $\mathrm{t}=140 \mathrm{~ms}$

Fig. 9 The level set profiles and the velocity fields during the condensation process under the normal gravity condition $(\mathbf{a}-\mathbf{i})$

Using the semi-implicit discretization method, the following equations can be obtained

$\frac{x^{n+1}-x^{n}}{\Delta t}=a \Delta^{\prime} x^{n+1}+\nabla^{\prime} \cdot\left(w^{n} \nabla^{\prime} x^{n}\right)-a \Delta^{\prime} x^{n}$

$\frac{y^{n+1}-y^{n}}{\Delta t}=a \Delta^{\prime} y^{n+1}+\nabla^{\prime} \cdot\left(w^{n} \nabla^{\prime} y^{n}\right)-a \Delta^{\prime} y^{n}$

Where $a=\max w^{n}$. Here $\Delta^{\prime}$ and $\nabla^{\prime}$ are the operators (the Laplacian and the gradient with respect to $(\xi, \eta)$. w is the monitor function.

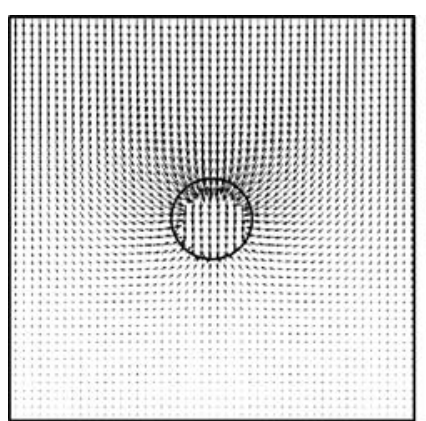

(a) $\mathrm{t}=1 \mathrm{~ms}$

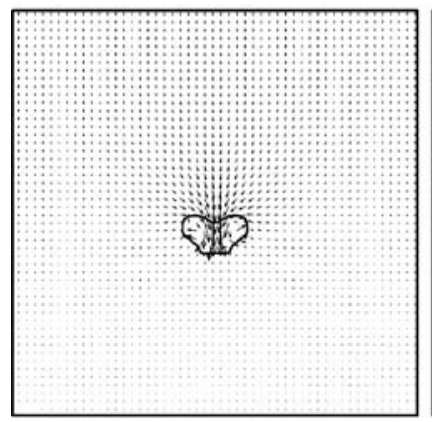

(c) $\mathrm{t}=40 \mathrm{~ms}$

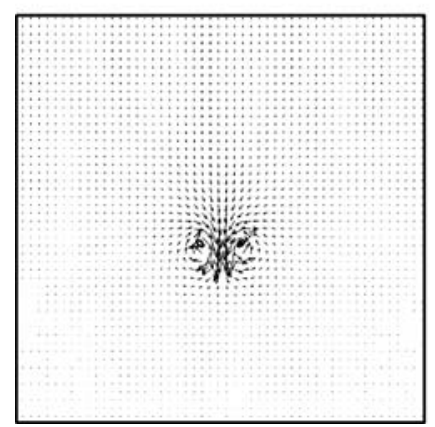

(e) $\mathrm{t}=80 \mathrm{~ms}$

Fig. 10 The level set profiles and the velocity fields during the condensation process under the microgravity $\left(10^{-2 *} \mathrm{~g}\right)$ condition $(\mathbf{a}-\mathbf{e})$

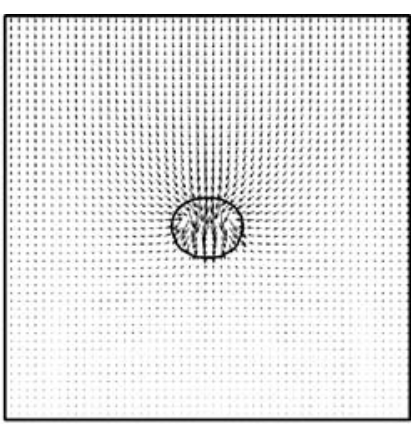

(b) $\mathrm{t}=20 \mathrm{~ms}$

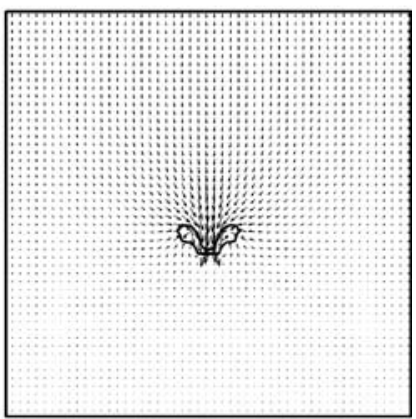

(d) $\mathrm{t}=60 \mathrm{~ms}$ 


\section{Grid Distributions}

Figure 8 shows the grid distributions at the time intervals of 0,20 and $40 \mathrm{~ms}$ under the normal gravity condition. The results show that the grid distributions in this paper can catch the interface continuously in space and time.

\section{Results and Discussion}

Figures 9,10 and 11 show the velocity fields and level set profiles during the condensation process of the single bubble, respectively, under the normal gravity conditions and the microgravity conditions. As shown in Fig. 9, 10 and 11, the volume of the bubble is kept

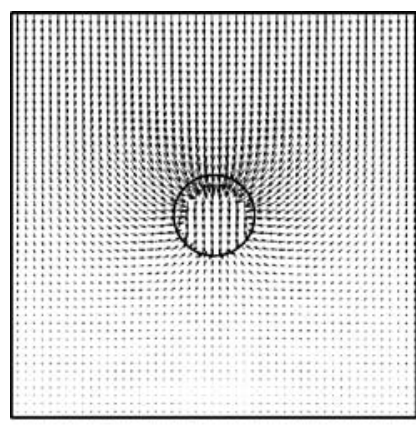

(a) $\mathrm{t}=1 \mathrm{~ms}$

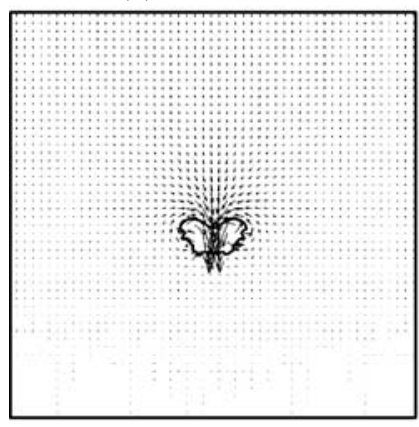

(c) $\mathrm{t}=40 \mathrm{~ms}$

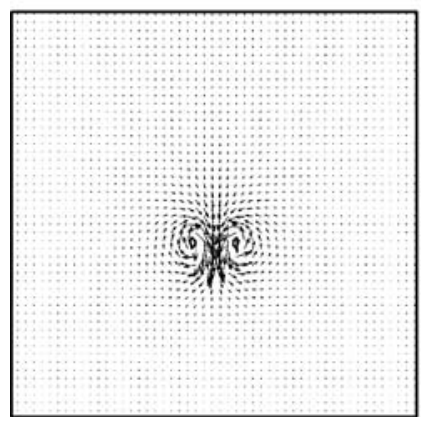

(e) $\mathrm{t}=80 \mathrm{~ms}$

Fig. 11 The level set profiles and the velocity fields during the condensation process under the microgravity $\left(10^{-4 *} \mathrm{~g}\right)$ condition $(\mathbf{a}-\mathbf{e})$ reducing until it disappear. Vortex appears on each side of the bubble, and the bubble will split into two small bubbles after a while. The whole condensation time is longer under the normal gravity than under the microgravity conditions.

In space engineering and applications, the condensation rate of the bubble is faster. Abnormal operating conditions may be more easily to be caused under microgravity conditions. The correct operation rules must be made to keep the flow from abnormal conditions.

\section{Conclusions}

A robust numerical method for simulation of two phases flows including mass and heat transfer under microgravity conditions is presented in this paper. The numerical results show that the method presented in this paper is more accurate than that used in the collocated grid. Single bubble condensed in the cold water under different level of gravity was simulated in this paper. The results show that the condensation process under the normal gravity condition is quite different from the condensation process under microgravity conditions. The whole condensation time is much longer under the normal gravity than under the microgravity conditions.

\section{References}

Ceniceros, H., Hou, T.Y.: An efficient dynamically adaptive mesh for potentially singular solutions. J. Comput. Phys. 172, 609$639(2001)$

Choi, S.K.: Note on the use of momentum interpolation method for unsteady flows. Numer. Heat Transf., A 36, 545-550 (1999)

Majumdar, S.: Role of underrelaxation in momentum interpolation for calculation of flow with nonstaggered grids. Numer. Heat Transf. 13, 125-132 (1988)

Miller, T.F., Schmidt, F.W.: Use of a pressure-weighted interpolation method for the solution of incompressible NavierStokes equations on a non-staggered grid system. Numer. Heat Transf. 14, 213-233 (1988)

Rhie, C.M., Chow, W.L.: A numerical study of the turbulent flow past an isolated airfoil with trailing edge separation. AIAA J. 21, 1525-1552 (1983)

Ryskin, G., Leal, L.G.: Numerical simulation of free-boundary problems in fluid mechanics. J. Fluid Mech. 148, 19-35 (1984)

Shklyar, A., Arbel, A.: Numerical method for calculation of the incompressible flow in general curvilinear co-ordinates with double staggered grid. Int. J. Numer. Methods Fluids 41, 1273-1294 (2003)

Wu, J.F., Dhir, V.K.: Numerical simulation of subcooled nucleate boiling by coupling level-set method with moving mesh method. Numer. Heat Transf. 51, 535-563 (2007)

Yuan, M.H., Yang, Y.H., Li, T.S., Hu, Z.H.: Numerical simulation of film boiling on a sphere with a volume of fluid interface tracking method. Int. J. Heat Mass Transfer 51, 1646-1657 (2008) 